Article

\title{
Sealing of Dry Particles with Grease-Filled Non-Contacting Sealing Systems
}

\author{
Florian Bosch * and Werner Haas \\ Institute of Machine Components, Department Sealing Technology, University of Stuttgart, \\ 70569 Stuttgart, Germany \\ * Correspondence: florian.bosch@ima.uni-stuttgart.de
}

Received: 30 June 2017; Accepted: 10 September 2017; Published: 11 October 2017

\begin{abstract}
There is no wear and no friction with non-contacting sealing systems. Because of this, there is in principle no lifetime-limit. However, this does not apply for grease-filled labyrinth seals. To seal against particles, the grease inside the gaps of the seal has to bind pollution particles and the grease-filled gap must be opened. If the surfaces of the grease layers in the gaps are saturated with particles, further particles penetrating the sealing system can no longer be bound. The seal must then be relubricated. Based on the actual state of the art, this work shows the latest results of experiments to enable designers to unerringly develop non-contacting sealing systems with grease-filled gaps to seal dry particles with reliable function. To that end, a lot of experiments were done to expand existing design guidelines. Additionally, new operating instruction were found to ensure permanent operation. A possibility to reduce the complexity of the sealing system is presented at the end of the present work.
\end{abstract}

Keywords: non-contacting sealing system; labyrinth sealing system; grease; particles; design rules

\section{Introduction}

Preventing sand or particles entering a housing is an important task in most technical applications. Otherwise, machine elements like bearings or gears can be damaged.

However, contacting sealing systems are often not suitable for sealing against particles as they would wear out too quickly. Non-contacting sealing systems are the solution to this problem. The two parts of the sealing system are separated by a small gap, so the parts with different speeds do not contact each other; because of this, there is no wear.

However, there is no standard sealing system that can meet all these requirements. For sealing against fluids, there are well researched non-contacting sealing systems, but the operating mechanisms that are used in those sealing systems cannot be used to seal against particles. For this, grease-filled labyrinth seals have been used for decades. However, there are no guidelines that ensure the reliable development and operation of those sealing systems, and there are no sealing systems ready for application.

So the question is, what has to be done to ensure a reliable non-contacting sealing system that seal particles for the whole lifetime of a machine? To solve this task, this article will show several results of numerous experiments that led to a better understanding of grease-filled labyrinth sealing systems. All experiments are based on design examples that can be found in the literature and the result of one research project done by Kümmel at the Institute of Machine Components at the University of Stuttgart.

The aim of this paper is to provide an overview of the state of the research on grease-filled non-contacting sealing systems to seal particles. For this reason, in addition to unpublished results, already published results [1-4] are shown. This approach appears to the authors as a reasonable means of providing the reader a complete and coherent overview on the research project. 


\section{State of the Art}

This section shows that there are a lot of design examples of existing sealing systems.

\subsection{Design Examples of Grease-Filled Labyrinth Sealing Systems}

Most design examples with non-contacting sealing systems were published by manufacturers of roller bearings as a part of design examples of the bearing point. Normally, there is no description of the functionality of the sealing system. Figure 1 shows an excerpt of an extensive collection of design examples of possible sealing solutions.

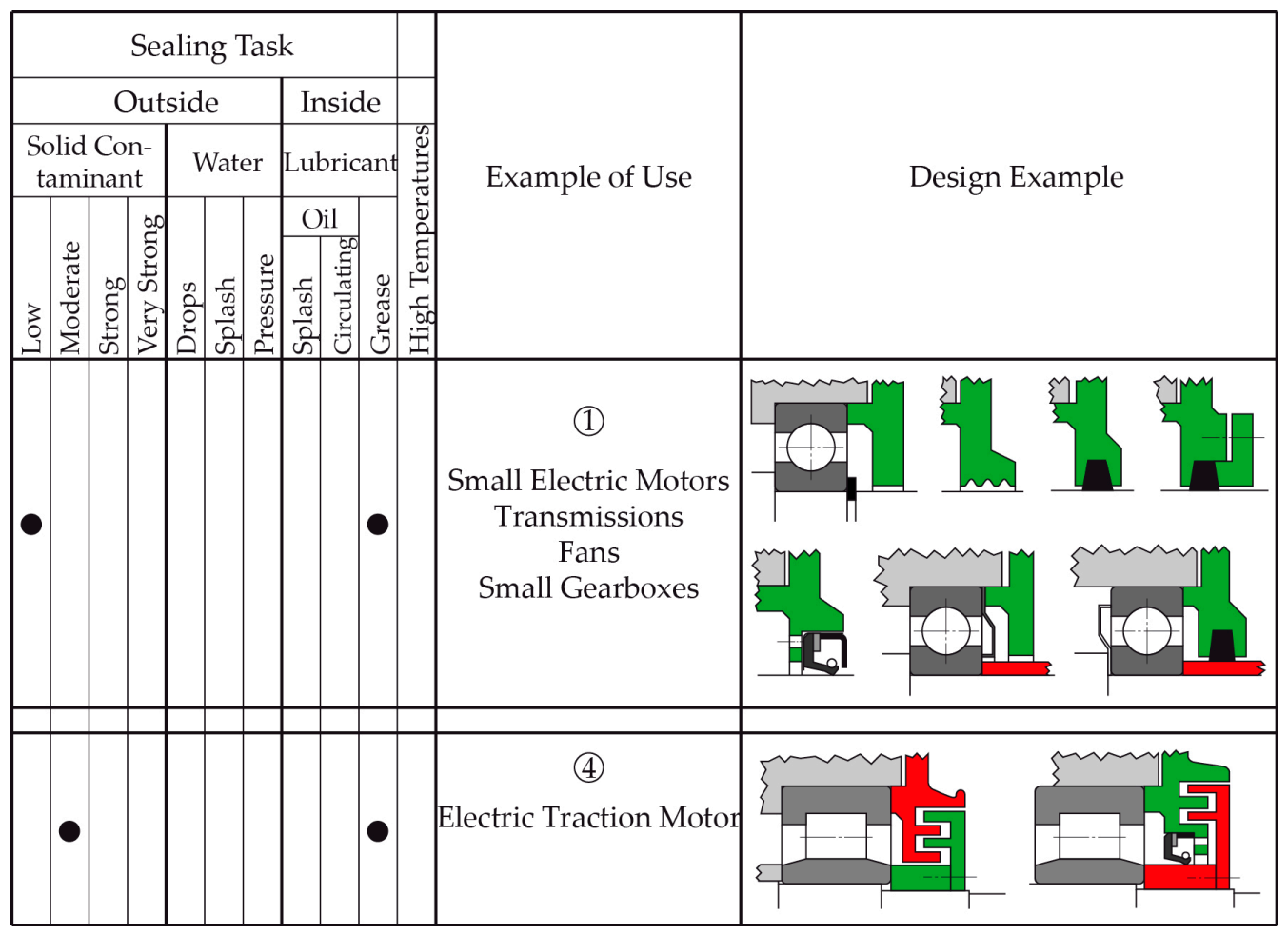

Figure 1. Excerpt from "typical examples of solution of sealing tasks" according to $[5,6]$.

In the right column, there are many different design examples ("Konstruktionsbeispiel") for a possible sealing solution. In the middle column, application possibilities are listed for the seals that are shown in the same line. The 11 columns on the left side specify the fluid or pollution for both sides of the seal, which can be sealed up with the design examples shown in the same line. As a summary of all the different design examples of non-contacting sealing systems with grease-filled gaps, Figure 2 shows the three different types.
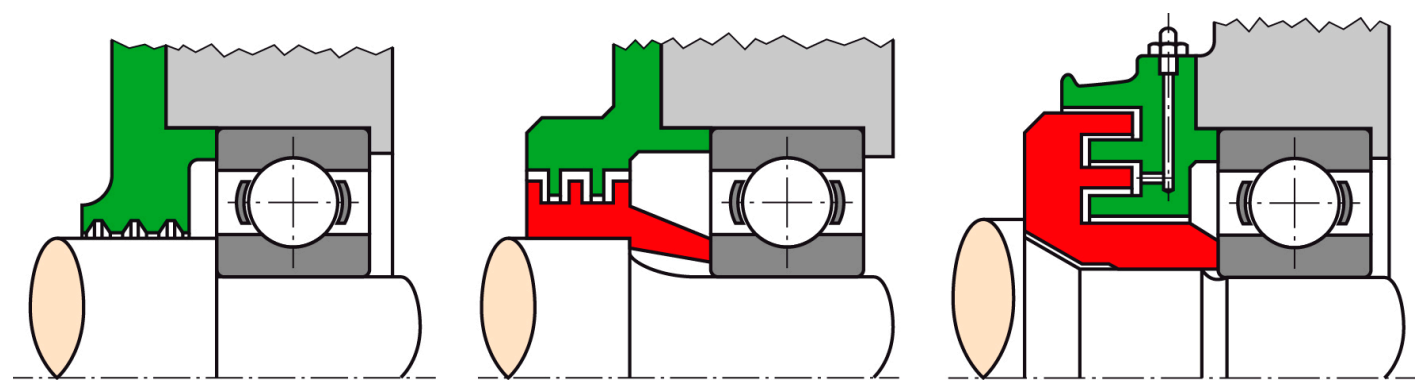

Figure 2. Excerpt from "typical examples of solution of sealing tasks" according to [7]. 
In the left figure of Figure 2, there is a simple axial gap. Optionally, the gap could be expanded by circumferential grooves that should hold the grease in the gap. The figure in the middle shows a radial labyrinth sealing system. In the right figure, there is an axial labyrinth sealing system. The labyrinth sealing systems are named by the longer gap. In the right example, the gaps in axial direction (horizontally in this example) are longer than gaps in radial direction (vertically in this example). The seal is therefore named the axial labyrinth sealing system.

In general, the disadvantage of such collections of different design examples is that the designer does not get any information as to which of the example seals is the best one for the application. If there is a decision for a design, the literature usually does not specify any dimensions. One exception is shown in Figure 3.

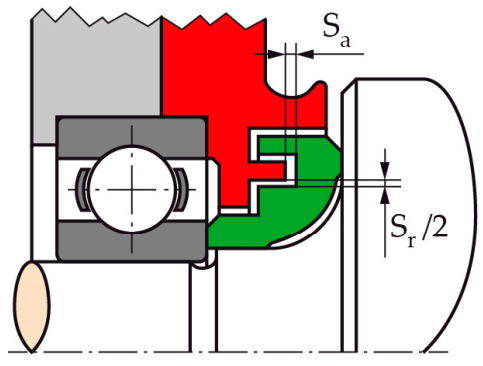

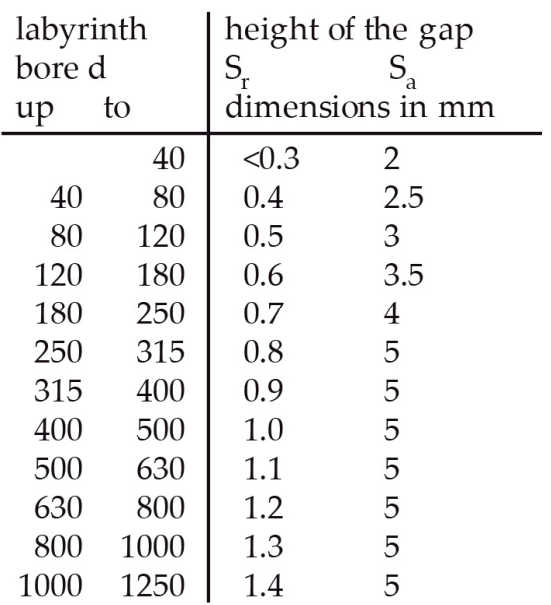

Figure 3. Recommended gap height of labyrinth sealing systems according to [7].

Figure 3 shows one of the few source where the designer of the sealing system can find dimensions of the gaps. It must be noted that the height of the gaps depends on the diameter of the bore of the sealing system: the greater the diameter, the greater the gap height. The second particularity is the way in which the height of the axial gap is specified: "S${ }_{r}$ " means the difference between the diameter of the rotating part and the static part of the walls limiting the gap.

\subsection{Grease-Filled Labyrinth Sealing System to Seal against Particles}

Because there is no further information about grease-filled labyrinth sealing systems, a research project was started at the Institute of Machine Elements at the University of Stuttgart. It was finished in 2010. The task of the project was to research grease-filled labyrinth sealing systems scientifically to get information about the basic functional mechanism and to generate design guidelines to ensure an unerringly design for reliable sealing systems.

Figure 4 shows an elementary part of a radial labyrinth sealing system. There is a rotating disc mounted on the shaft. It is covered by two transparent static discs. The gap between those three discs is completely filled with grease. During the experiments, the grease inside the gaps could be observed through the transparent discs. The experiment with this sealing system was divided into two parts. In the first part, colored grease was pumped into the gap with low pressure. In the second part of the experiment, the colored grease was pumped into the gap with high pressure. The track off the colored grease was observed through the transparent discs.

If the colored grease was pumped into the gap with low pressure, there was a track like a spiral starting from the bore inward to the shaft. If the colored grease was pumped into the gap with high pressure, the colored grease spread outward. Tracking the colored grease in a cross section of the sealing system, one can find a circular flow of the grease going outward at the rotating disc and going back to the shaft just in front of the static transparent disc (see Figure 4). 


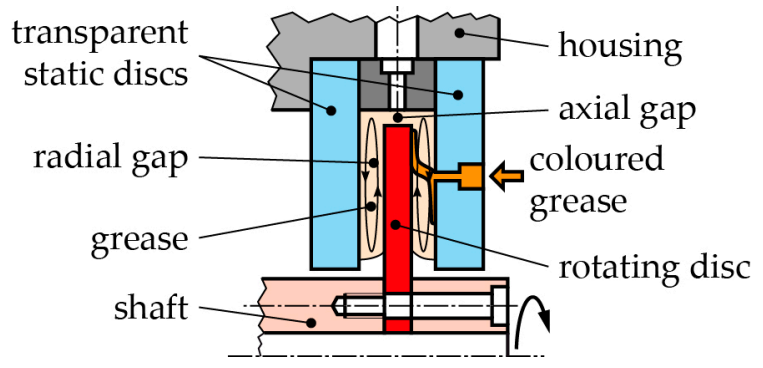

Figure 4. Circulating flow of grease in completely filled gaps according to [8].

In a second step, axial labyrinth sealing systems were sprayed with particles for two hours. After the experiment, the sealing systems were disassembled very carefully. Samples of the grease were taken and were investigated with a microscope.

Figure 5 shows the degree of pollution of the grease inside the gaps of a grease-filled labyrinth sealing system at seven different sampling positions. The sampling positions are shown in the small figure in the upper right corner. The experiment was done at six different shaft speeds.

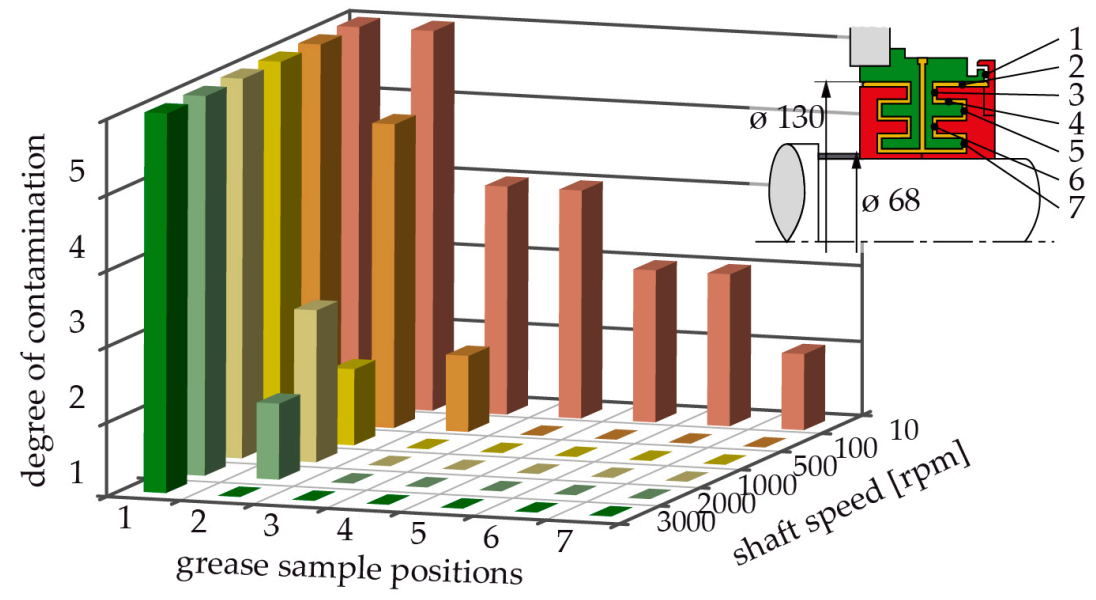

Figure 5. Contamination of the grease in an axial labyrinth sealing system with grease-filled gaps polluted for two hours with particles according to [8].

At $10 \mathrm{rpm}$, the grease was polluted with particles at all seven sampling positions. The contamination was greatest at the beginning of the sealing system (positions 1 and 2). The longer the distance from the entrance of the sealing system, the lower the contamination of the grease. In the middle of the sealing system (position 7), the grease pollution was very low.

Increasing the shaft speed, the particles seem not to be able to penetrate the sealing system as at lower shaft speed. For example, at $100 \mathrm{rpm}$, there was no more contamination of the grease at position 4. With further increase of the shaft speed, the grease was only polluted with particles at the very beginning of the sealing system. One can therefore say that the shaft speed has to rise over a certain value before the particles can be sealed with grease-filled labyrinth sealing systems.

Figure 6 shows the measurement result of the friction torque. It is the result of the same experiment in Figure 5. In Figure 6, one can see the measured torque against time. The experiment lasted two hours. During a short time at the beginning of the experiment, the torque decreased very fast. At $10 \mathrm{rpm}$, the average torque during the whole experiment was about $0.4 \mathrm{Nm}$. At $10 \mathrm{rpm}$, the particles could penetrate to the middle of the sealing system (position 7 in Figure 5). At $100 \mathrm{rpm}$, the torque decreased, although the shear stress increased compared to $10 \mathrm{rpm}$. With further increasing of the shaft speed, the measured torque decreased to $0 \mathrm{Nm}$. At those shaft speeds, the particles could only contaminate the first two gaps. Therefore, particles can be sealed with this sealing system if the torque is $0 \mathrm{Nm}$. 


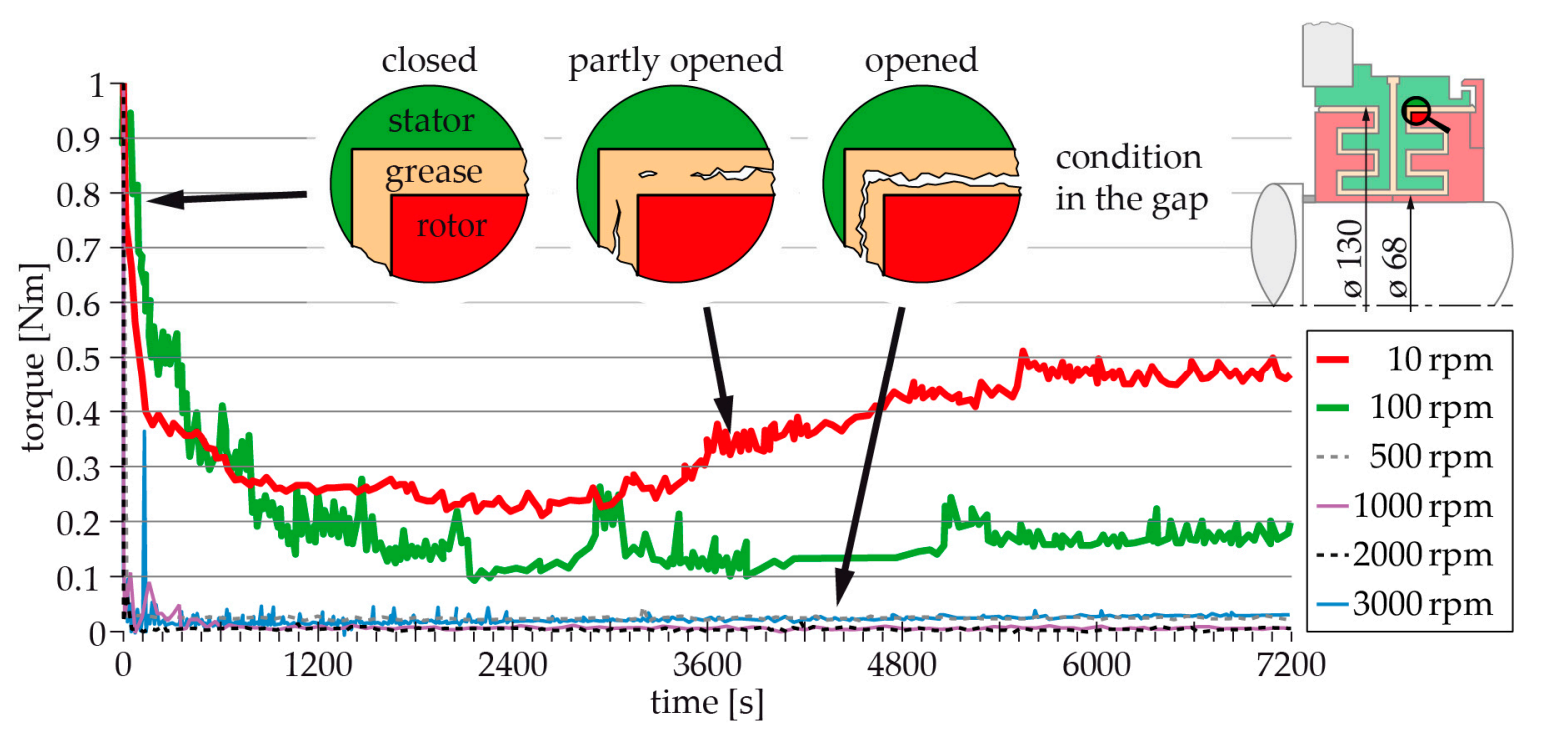

Figure 6. Friction torque of an axial sealing system grease A, NLGI 2 according to [8].

To clarify why the torque decreases to $0 \mathrm{Nm}$ at the same shaft speed to seal the particles, an experiment with transparent walls was done (as in Figure 4). In this experiment, one could see that the grease-filled gap opened. An "opened gap" means that there are two layers of grease adhering at the rotating and the static part of the sealing system. Those layers of grease are separated by a thin layer of air and do not touch each other. Because of this, there is no shear stress in the grease: only the air gets sheared. This is the explanation of the decreasing torque shown in Figure 6. At $10 \mathrm{rpm}$, nearly the whole gap is completely filled with grease. If the shaft speed increases, there are some areas in the gap that are opened. At $500 \mathrm{rpm}$, the whole gap opens.

This means that if the gap is completely opened, there is no flow of the grease inside the gaps that can transport bound particles through the sealing system. If the gap is closed, it is a function of time until there are particles at the other sides of the sealing system; these particles are transported through the sealing system by the grease flow inside the gaps. Furthermore, if the gaps are completely filled with grease (closed), all the particles that entered the sealing system will be held by the grease, and the pollution of the grease rises. If the gap is opened and the shaft is rotating, particles can be pushed out of the sealing system by centrifugal forces. Particles will not be bound by the grease if the surface of the grease layer is saturated with particles in the very beginning of the gap. This leads to the basic prerequisite that the grease-filled gaps must be opened to seal particles with grease-filled non-contacting sealing systems [9].

The grease inside the gaps binds particles that pass the entrance area of the seal and get into the main part of the sealing system. If there is no grease to bind those particles mechanically, the particles will be able to pass the sealing system in a very short time. This is based on the principle functionality of non-contacting sealing systems without a blocking liquid $[10,11]$.

As a further result of the Kümmel research project, design guidelines for grease-filled labyrinth sealing systems were developed, which are shown in Figure 7. The dimensions in the tables right next to the belonging figures are given as a range according to the height of the gap $h$.

The upper figure shows the covered entrance area of the labyrinth sealing system. It is characteristic for all non-contacting sealing systems and has to prevent particles and fluid from getting into the gap of the sealing system. Right next to it, there is the table with all necessary dimensions. The figure and table below show all necessary dimensions of the labyrinth sealing system. In the lower area, the table shows the operating parameters that were necessary to guarantee an opened gap in the Kümmel experiments. 


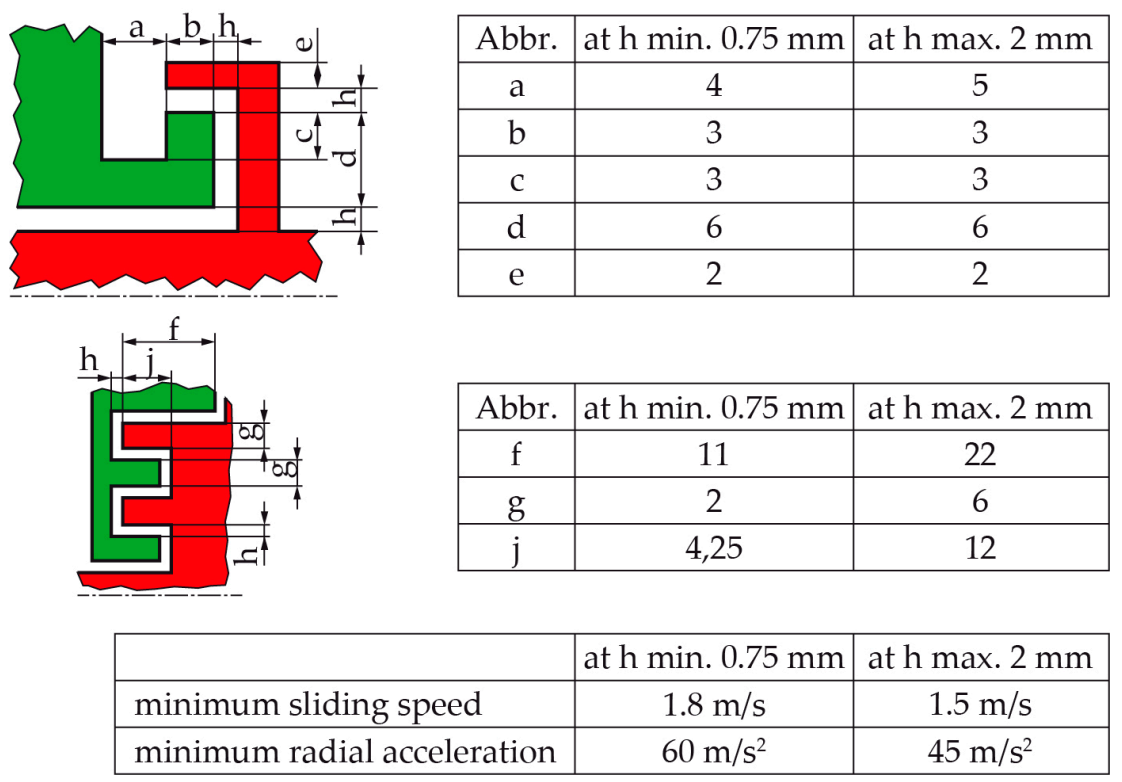

Figure 7. Design guidelines for grease-filled labyrinth sealing systems according to [8].

In addition to the results of Kümmel shown above, there are some works on the grease in small pockets of contacting sealing systems, e.g., [12,13]. Besides the velocity profile of the sheared grease, the motion of particles bound by the grease was observed. It was concluded that particles in the grease will move radially outward if the grease is sheared. The radial migration depends on the height of the grease layer. Depending on the radial migration, the probability to reduce contaminants by relubrication is affected. However, those results are only valid for contacting sealing systems, because during the experiments, the observed pocket was completely filled with grease. The results of Kümmel showed that non-contacting sealing systems can only be used to seal particles if the grease-filled gap is opened. This means there are two layers of grease adhering at the rotating and static parts of the sealing system, which are separated by a layer of air. Those conditions are characteristic for non-contacting sealing systems with grease-filled gaps. Because of this, there is no flow velocity of the grease.

\section{Relubrication of the Sealing System}

The results of Kümmel displayed in Figure 5 show that sealing particles with grease-filled labyrinth sealing systems is possible. However, if the surface of the grease layers inside the gaps is saturated with particles, the sealing system has to be relubricated with fresh grease. It has to replace the polluted grease to maintain the function of the sealing system.

The literature does not provide any data on how much grease is needed to relubricate the sealing system. The only indication is that in the past, grease-filled labyrinth sealing systems were often used to seal roller bearings. There are instructions for the relubrication of roller bearings. For example, the companies NTN/SNR, SKF, and INA/FAG recommend the bearing be relubricated with approx. $20 \%$ to $40 \%$ of the available volume inside the bearing [14-16]. The lower value is recommended if the bearing is relubricated directly into the middle of the bearing. The upper limit is recommended if the bearing is relubricated beside the bearing.

In the experiments, the bore to relubricate the system runs into the system directly next to the bearing, as shown in Figure 8. The idea for the experimental setup is derived from real application. It consists of a standard roller bearing that is sealed on both sides with a grease-filled labyrinth sealing system. The sealing systems are made according to the guidelines developed by Kümmel [8]. For the experimental setup, a standard roller bearing $6213\left(65 \times 120 \times 23 \mathrm{~mm}^{3}\right)$ was used. According to [15,16], the recommended amount of grease to relubricate the bearing is $m=14 \mathrm{~g}$. 


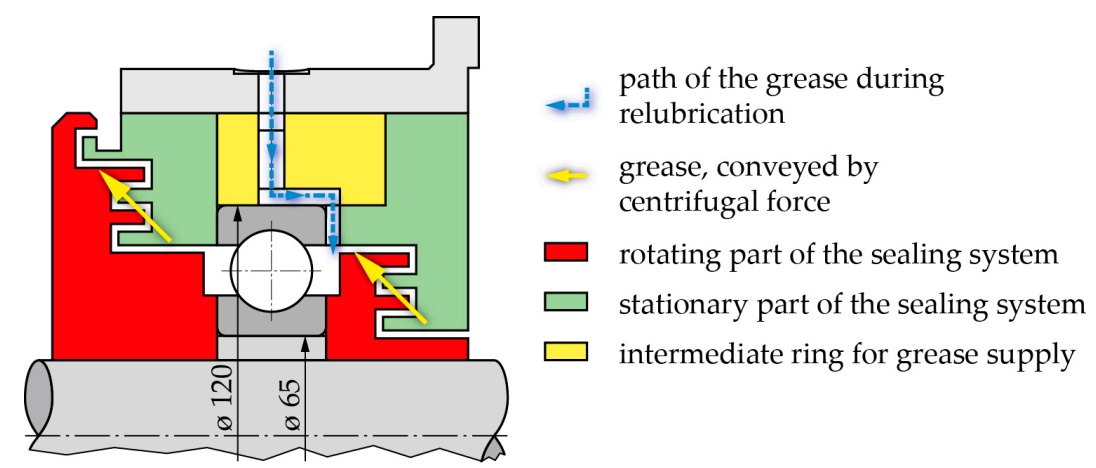

Figure 8. Experimental setup.

\subsection{Preliminary Experiment}

The aim of this preliminary experiment was to find the shaft speed at which the gap is open. For this experiment, the bearing was completely filled with grease, and the shaft speed was increased in steps of $100 \mathrm{rpm}$ up to $3000 \mathrm{rpm}$. The friction torque caused by the bearing and the sheared grease was measured during the entire experiment. This is shown in Figure 9. The measurements can be divided into four sections:

1. The offset is caused by a low shaft speed. The torque increases almost linearly with increasing shaft speeds of up to $1000 \mathrm{rpm}$. The torque is caused by the friction of the bearing. In addition, the grease is displaced by the bearing and gets into the gaps of the sealing system with increasing speed, where it is sheared.

2. Between $1000 \mathrm{rpm}$ and $1700 \mathrm{rpm}$, the friction torque appears not to depend on the shaft speed. This is because the grease is nearly completely displaced by the bearing. There is no new grease getting into the gaps. In addition, some gaps begin to open.

3. At more than $1700 \mathrm{rpm}$, the friction torque decreases with increasing shaft speed. This is caused by the opening gaps.

4. At more than about $2000 \mathrm{rpm}$, the friction torque increases slowly with increasing shaft speed. It corresponds to the friction torque of a bearing that is lubricated with a minimal amount of grease. It follows that all gaps are open, and there is no sheared grease left in the gaps.

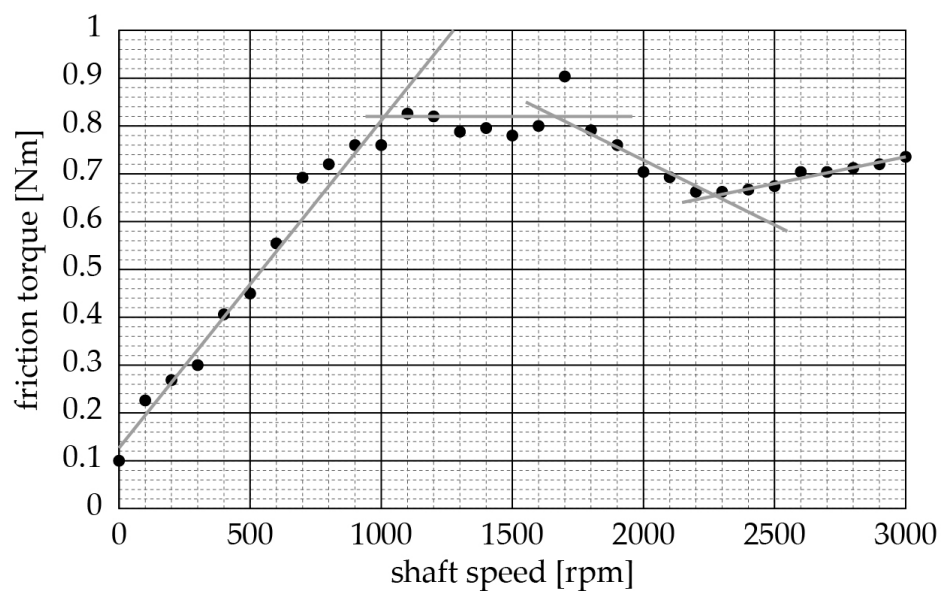

Figure 9. Friction torque during the preliminary experiment to determine the necessary shaft speed for an opened gap.

To summarize the preliminary experiment, a shaft speed of $2000 \mathrm{rpm}$ is sufficient to ensure that the grease is distributed inside the experimental setup and to guarantee the opening of the 
grease-filled gap. The circumferential speed at the average diameter of the bearing $\left(\varnothing_{\text {bearing }}=92.5 \mathrm{~mm}\right.$ and $n=2000 \mathrm{rpm}$ ) is then $u=9.69 \mathrm{~m} / \mathrm{s}$.

\subsection{Test Procedure}

During the experiments shown in this paper, two different greases were used:

- $\quad$ NLGI 0/00-Klüber ISOFLEX Topas NCA 5051 [17];

- $\quad$ NLGI 2-Klüberbio M 72-82 [18].

Three different groups of experiments were done. They differ in the prepared lubricating grease, which was filled into the sealing system just before the experiments started. The prepared grease was:

- $\quad$ Fresh grease;

- $\quad$ Aged grease;

- $\quad$ Grease polluted with particles.

To distinguish the grease to be replaced during the experiments from the relubricated grease, the prepared grease was colored blue. The rheological properties of the grease were not affected by the coloring. To age the grease, it was stored at $120^{\circ} \mathrm{C}$ in an oven for a few hours until the grease lost about $35 \%$ of its base oil. According to Bartz [19], grease is capable of lubricating a bearing until it loses about $50 \%$ of its base oil. The grease used in the experiment was like grease that should be replaced very soon, but it was still usable. In the third part of the experiment, the grease was polluted with a specific amount of particles.

Before the start of the experiment, the experimental setup was completely cleaned from any grease. Then, the bearing was completely filled with fresh grease. The prepared grease was filled into a groove of the outer (left-hand side) sealing system. The prepared grease was not allowed to get into the bearing. Finally, the experimental setup was mounted onto the test bench.

The first section of the experiment was a grease distribution cycle. Then, the experimental setup was relubricated with fresh grease, followed by another grease distribution cycle. The last two sections (relubricating and grease distribution cycle) were repeated several times.

The friction torque was measured with a lever and a force sensor. The stationary part of the sealing system was mounted in an aerostatic bearing. The friction torque could thus be measured very accurately without friction losses.

After the experiment, the experimental setup was dismounted from the test bench. Finally, the outer sealing system, which was filled with prepared grease before starting the experiment, was opened to take grease samples at different positions in the sealing system. To document the result of the relubrication cycles, pictures of the cross-section of the grease inside the grooves were taken. To make the cross-section visible, grease had to be locally removed. Figure 10 shows the building of a cross-section inside the grooves of the dismounted sealing system (rotor or stator). In the photograph on the right-hand side, one can see the old (blue colored) grease in the bottom of the groove. The upper layer of grease is already beige colored. This means it is relubricated grease.

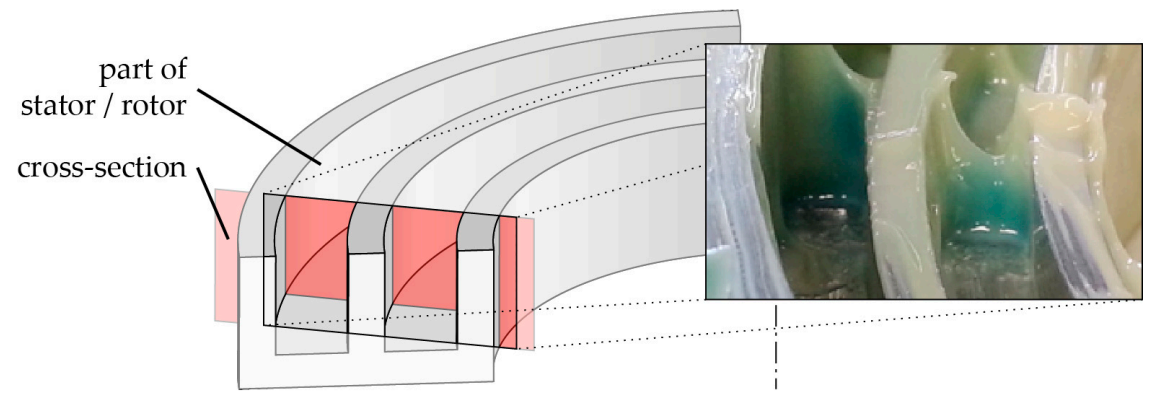

Figure 10. Schematic illustration of sample documentation after experiments with colored grease. 


\subsection{Results of Relubricating Experiments}

Because of the special behavior of grease as a non-Newtonian fluid and the fact that the transition between the layers of old and fresh grease is smooth, the results of the relubricating experiments can only be documented qualitatively, as shown in Figure 11.

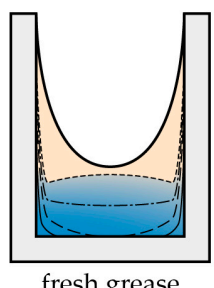

fresh grease

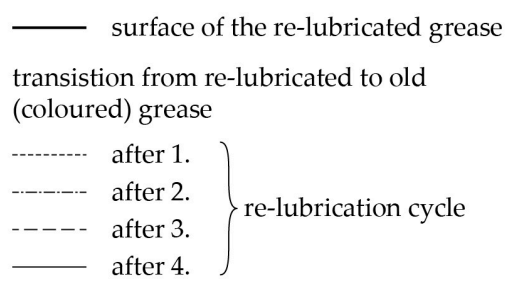

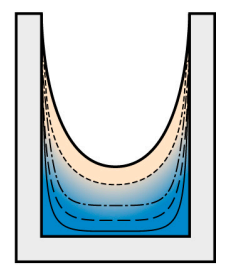

aged grease

Figure 11. Qualitative documentation of the relubrication results.

The results of the relubricating experiments with fresh grease as prepared grease are shown on the left-hand side of Figure 11. The relubricated grease is colored in orange at the surface of the grease. The different dotted lines represent the transition between the relubricated fresh grease on the top and the old, colored grease at the bottom of the groove. One can see that even after the fourth relubricating cycle, there is still a small amount of old grease left in the corners of the groove.

At the right-hand side of Figure 11, the result of the relubricating experiments with aged grease are shown. Before the experiment, the grease lost a significant amount of its base oil and is therefore much thicker than the new grease. One can see that the amount of grease that was replaced by relubricating the experimental setup was much smaller compared with the result of the experiments with fresh grease (left-hand side). After the fourth relubricating cycle, there was still a layer of old grease adhering on the walls of the groove. Even with further relubricating cycles, the layer of old grease could not be removed completely.

If polluted grease was filled into the groove of the sealing system before the experiment, the results of the experiments would be quite different. The result of the relubricating cycles depends on the position of the gap: whether the inner or outer wall of the gap is rotating. Figure 12 shows three samples of polluted grease taken from different positions.

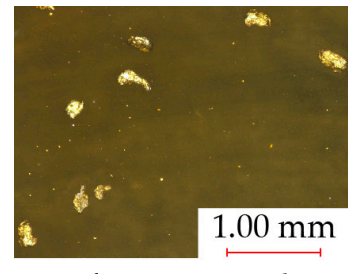

reference sample

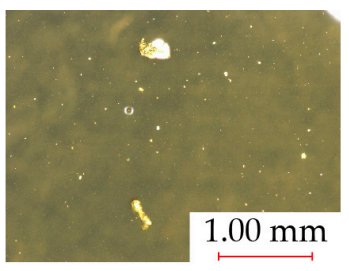

from stator

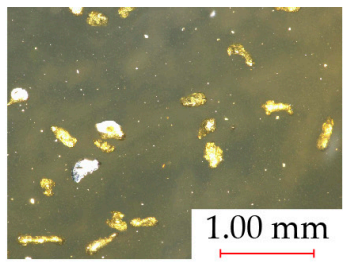

from rotor

Figure 12. Example of polluted grease taken from the prepared grease before the experiment starts and from the stator and rotor after the experiment.

The first sample was taken from the prepared grease before the experiment was started. It is the reference sample for the following interpretation of the relubricating results. The second sample (middle of Figure 12) was taken from the grease adhering at the static part of the sealing system after the experiment. The third sample on the right-hand picture was taken from the grease adhering at the rotating part of the sealing system.

It can be seen that the pollution largely changed. At the static part of the sealing system, the particles could be removed from the gap by relubricating the experimental setup. At the rotating part of the sealing system, the situation was much worse. The concentration of particle bound by the grease after the experiment was much larger than before the experiment started. 
To explain this phenomena, one must know that the samples shown in Figure 12 were taken from a gap where the outer wall was rotated during the experiment. If the inner wall was rotated during the experiment, there were no particles left in the grease after the experiment.

Figure 13 illustrates the reason for the quite different results of the relubricating cycles that depend on the position of the gap where the sample was taken from. The complete sealing system is shown on the left-hand side of the figure. The enlarged details are shown in the figures on the right-hand side.

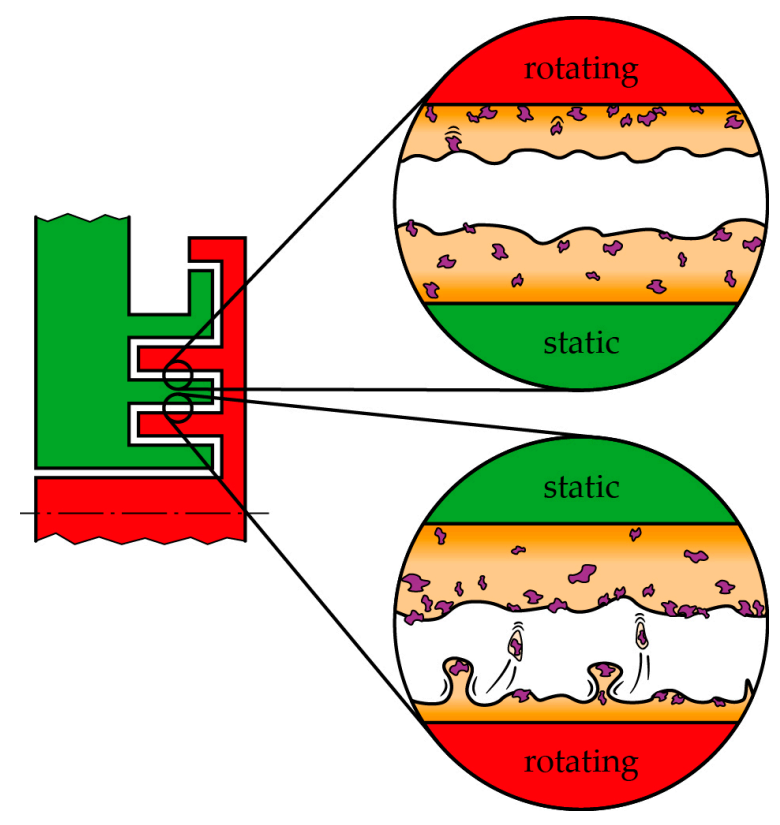

Figure 13. Movement of particles in the gap caused by centrifugal force.

In general, if the wall of the gap is rotating, there is a centrifugal force acting on the grease adhering at the wall and the particles bound by the grease. If the wall is static, there is no centrifugal force on the grease adhering at the wall or on the particle bound by the grease.

In the lower gap, the inner wall of the sealing system rotates. Because of this, there is a centrifugal force acting on the grease adhering at the wall and the particles bound by the grease. Therefore, the particles will move radially outward to the grease layer adhering at the static part. In the grease layer at the static part, the moved particles settle at the surface of the static grease layer. There, no centrifugal force is acts on the grease and the particles. If there is enough time, all particles move to the static part of the sealing system.

In the upper gap, the inner wall of the sealing system is at rest. Because of this, there is no centrifugal force, and the grease and the particles do not move. The outer wall of the gap rotates, so there is a centrifugal force acting on the grease and particles. This leads to a movement of the particles to the outside, too. In this case, they cannot move to another grease layer because there is a wall that limits the gap. This is the reason why the particles move to the outer wall of the gap.

\subsection{Reduction of Complexity of the Sealing System}

Combined with the results of Figure 11, where the grease adhering directly in front of the walls cannot be removed by relubricating the experimental setup, the results shown in Figures 12 and 13 lead to the conclusion that there are gaps that can easily be cleaned by relubricating the system, and there are gaps where particles cannot be removed by relubricating. In fact, if the outer wall of the gap rotates, particles can hardly be removed by relubricating. This means that particles are not allowed to get into a gap where the outer wall is rotating. Because gaps with a rotating outer wall and a rotating inner wall alternate, all gaps coming after the first gap with a rotating inner wall are wasted because particles 
that get into those gaps cannot be removed completely. This led to a great potential for simplification of the sealing system. This is shown in Figure 14.
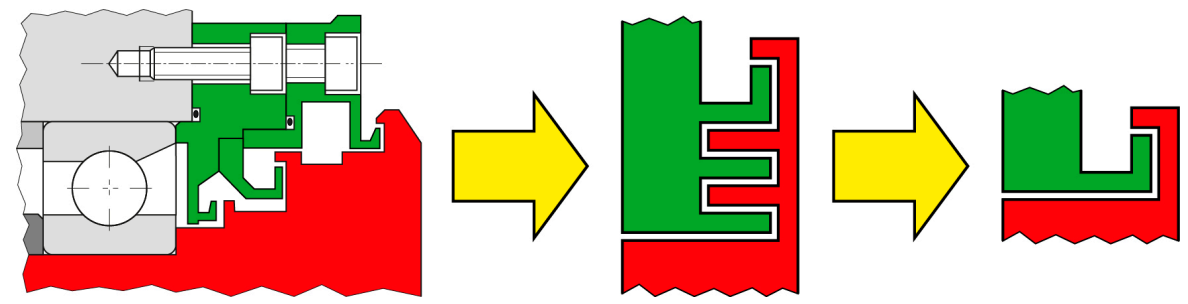

Figure 14. Reduction of complexity of the sealing system caused by the recognition that only each second gap can be cleaned of particles by relubrication.

On the left side in Figure 14, there is an example of a very complex non-contacting collecting labyrinth sealing system like it is used in machine tools. Normally, it is only used to seal water or cooling fluid mixed with particles, but it is a very good example of a complex sealing system. In the middle, there is a labyrinth sealing system with grease-filled gaps according to the design guidelines of Kümmel [8]. It is much less complex compared with the non-contacting collecting labyrinth sealing system on the left-hand side. The great step is shown in the last figure on the right. The sealing system only consists of a covered entrance area followed by a long axial gap. This sealing system is very easy to design and cheap to manufacture, but the reliable function of this seal has to be experimentally demonstrated. They are shown in the following section.

\section{Functional Tests with the Optimized Sealing System}

Figure 15 shows the sealing system that was build according to the results explained above. The sealing system only consist of a covered gap entrance followed by a long axial gap. The axial gap is about $20 \mathrm{~mm}$ long. The sealing system has a bore to relubricate the sealing system. This bore runs into the gap on the side facing away from the particles that were sprayed onto the sealing system from the right side.

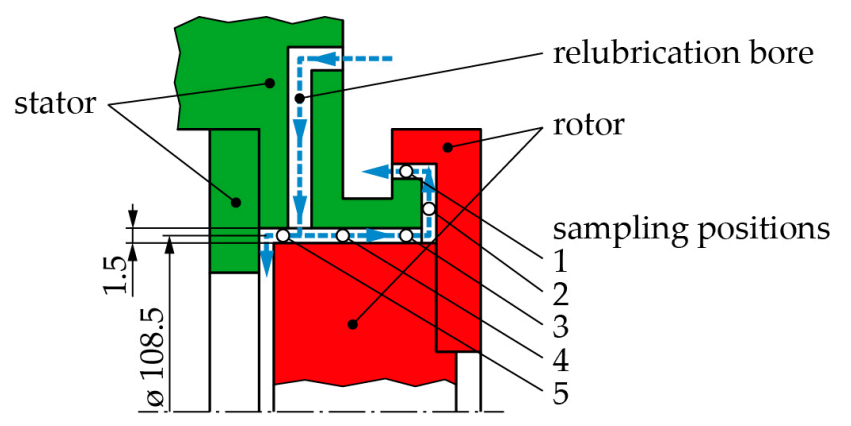

Figure 15. Experimental setup to investigate the function of the optimized sealing system.

\subsection{Test Procedure and Evaluation Method}

At the beginning of the experiment, the sealing system was filled with fresh grease and mounted at the test bench. The basic cycle of the experiments, which is shown below, was repeated several times.

- $\quad$ Grease distribution run to ensure a opened gap; shaft speed is $n=500 \mathrm{rpm}$;

- Contamination of the sealing system by spraying particles directly on entrance area of the sealing system normally for $10 \mathrm{~min}$ (other duration are given in the figures); shaft speed normally is $n=500 \mathrm{rpm}$;

- $\quad$ Relubrication of the sealing system with $m=14 \mathrm{~g}$, duration is $t=120 \mathrm{~s}$. 
After the last contamination period, the system was not relubricated. The sealing system was carefully dismounted from the test bench directly after the last contamination period. To evaluate the pollution of the grease inside the sealing system after the experiments, four samples of the polluted grease distributed at the circumference were taken at the five positions, which are marked in Figure 15. For each sample, three pictures were taken. The pollution of the grease at one position is the average of the result of the 12 pictures (four samples taken at the circumference, three pictures for each sample).

During the experiment, the particles were sprayed with a rotating plate directly to the entrance area of the sealing system. Quartz sand was used as particles, and $42.4 \mathrm{~g} / \mathrm{min}$ were sprayed onto the sealing system, which was $2.54 \mathrm{~kg} / \mathrm{h}$.

The measurement was done with a Keyence VHX 1000D microscope (Keyence GmbH, German Office: Siemensstraße 1, D-63263 Neu-Isenburg). The measuring area was $4.31 \mathrm{~mm}^{2}$. To count particles in the polluted grease, a layer of grease was observed with the microscope. The thickness of the grease layer was $1 \mathrm{~mm}$. However, only particles at the surface of the grease layer could be observed because the grease was almost not transparent. Because of this, the amount of particles shown in the following figures are not related on the volume of grease. The method used was nevertheless allowed because only results at the same conditions and done with the same procedure were compared. The particles had to be counted manually. An automated process was not possible because the contrast between the colors of the grease and the particles was too bad.

\subsection{Experiments with Constant Pollution}

In the first step, the sealing system was constantly polluted with particles. The sealing system was not relubricated. The experiment was done with two different greases (NLGI 0/00 and NLGI 2-grease). The result of the experiment is shown in Figure 16. The average amount of particles in the sample is shown against the duration of the experiment. The amount of particles was evaluated as the average of all taken samples.

Figure 16 shows that the particles penetrate the gap mainly during the first $30 \mathrm{~min}$ of the experiment. After this period, it seems that no further particles got into the gap. According to these results, after a period at the beginning of the experiment, the increase in pollution with time was very low (tendencies shown by the linear curve fitted lines) if the entrance area of the sealing system was designed in the best way, as in Figure 15. Figure 16 also shows that the amount of particles that enter the sealing system may vary significantly. This may be caused by the special behavior of lubricating greases and must be taken into account during the design process.

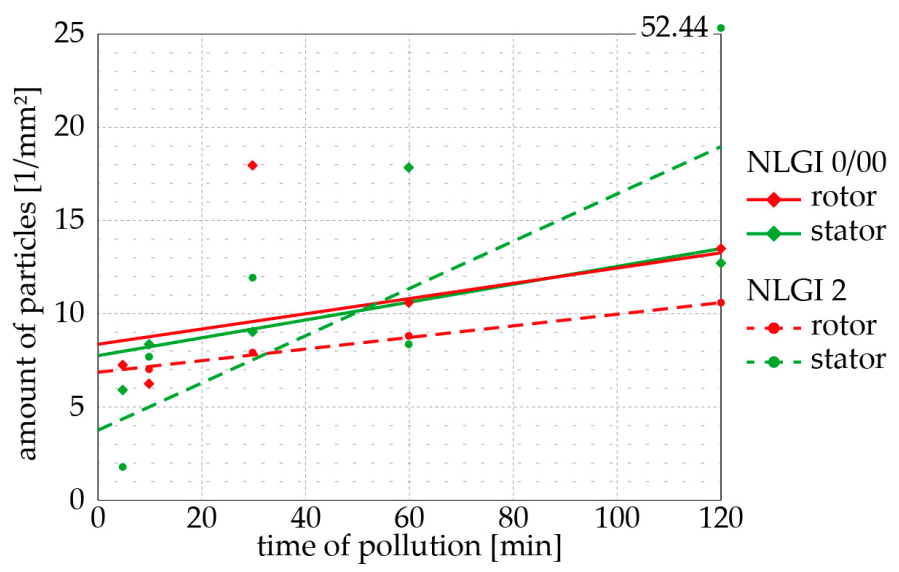

Figure 16. Sum of the amount of particles over all positions; constant pollution for different time; two different greases; $n=500 \mathrm{rpm}$; no relubrication; lines are linear curve fitted.

It was further found that there is no difference between the results of the experiments with different greases. If the grease filling gap is completely opened (prerequisite for a working seal), the 
consistency or type of grease does not affect the amount of particles that get into the sealing system. However, the shaft speed to get an opened gap is affected by the grease.

\subsection{Pollution of the Grease at Different Positions}

To see if the sealing system works reliably, the pollution of the grease must be evaluated at several positions. This is shown in Figure 17. Here, the amount of particles that were detected at the respective sampling position is shown against the sampling position.

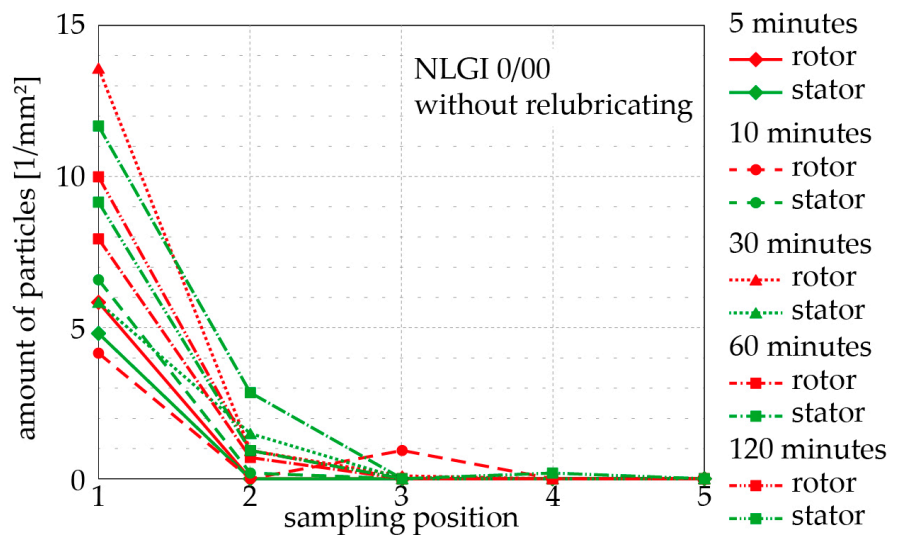

Figure 17. Pollution of the grease at several positions; constant pollution for different time, NLGI $0 / 00$-grease; $n=500 \mathrm{rpm}$; no relubrication.

At position 1, the grease is strongly polluted with particles. This was expected because position 1 is located directly at the entrance area of the sealing system. At position 2, there are only a few particles bound by the grease. Starting with position 3, the grease was completely clean. The few particles that were detected at positions 3 and 4 presumably fell into the gap when the sealing system was disassembled. With the NLGI 2-grease, the same result was achieved: only the grease in the entrance area of the sealing system was polluted with particles, and the grease inside the long axial gap was clean.

\subsection{Experiments with Relubricating}

Figure 18 shows the results of the evaluated pollution of the grease after experiments with one to five relubrication cycles at several sampling positions. The system was relubricated at $500 \mathrm{rpm}$, which is the shaft speed where the gap was reliably opened.

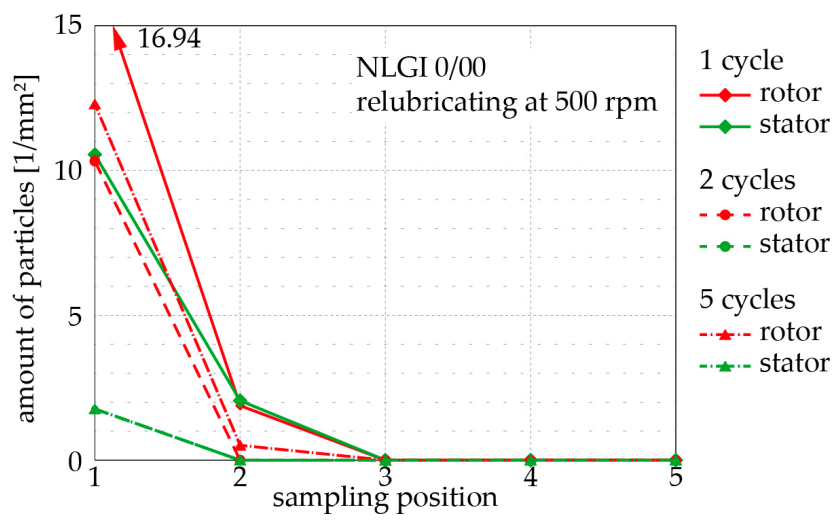

Figure 18. Pollution of the grease at several positions; $n=500 \mathrm{rpm}$; several times constant pollution (10 min each); relubrication at $n=500 \mathrm{rpm}$; NLGI 0/00-grease. 
The results shown in Figure 17 are similar to the pollution of the grease after experiments with relubricating the system, which are shown in Figure 18. At position 2, the grease was only very low polluted by particles, too. The grease in the complete axial gap (positions 3 to 5) was free of particles. Figure 18 also shows that the pollution of the grease decreases with the increasing number of relubricating cycles, even though there was a period of $10 \mathrm{~min}$ at the end of all experiments during which particles were sprayed onto the sealing system.

This is because excess grease from the relubrication cycles gets out of the sealing system. It remains partly at the entrance area out of the sealing system and forms a grease collar. This grease collar also binds particles. It forms a very narrow gap to the rotating part of the sealing system. This narrow gap is the reason why so few particles can enter the sealing system after a few relubrication cycles.

\subsection{Defining Two Functional Areas of the Sealing System}

The result of the experiments showed that only the grease in the entrance area of the sealing system was polluted with particles. The grease inside the axial gap of the sealing system was free of particles. Because of this, two functional areas can be defined: one area to seal against particles, and one area to relubricate the sealing system. Figure 19 shows the sealing system and the defined functional areas.

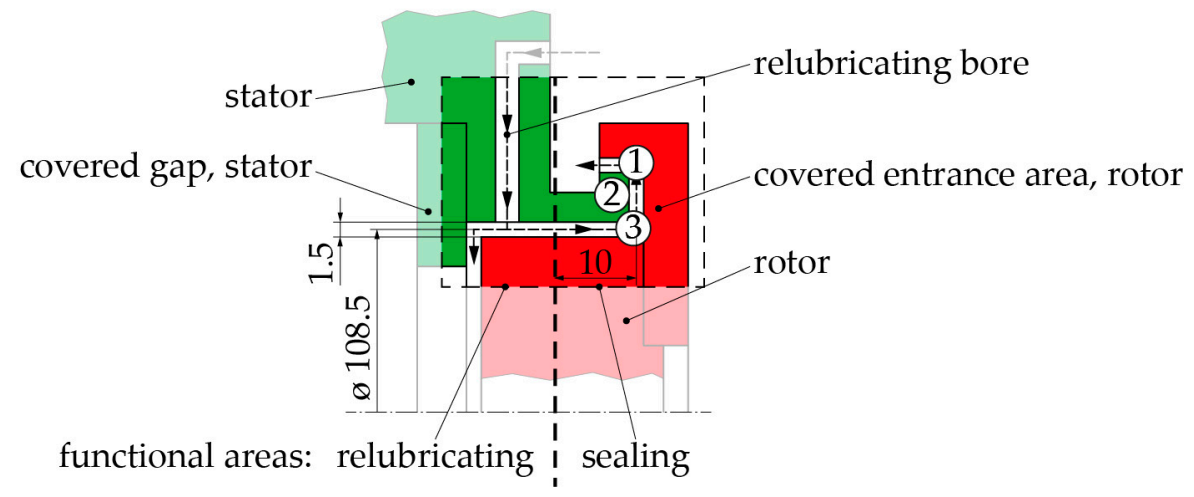

Figure 19. Definition of two functional areas of the sealing system: sealing against particles, and relubricating with fresh grease.

Because after the experiment there were no particles detected at position 3, two functional areas can be defined. The first functional area has the task to seal against particles. The second functional area is used to relubricate the sealing system with fresh grease. The functional area for sealing against particles contains the covered gap in the entrance area (position 1 in Figure 19) of the sealing system, the first radial gap (position 2), and the first half of the long axial gap (position 3). The second functional area to supply the sealing system with fresh grease uses the second half of the axial gap (position 5) where a bore to relubricate the sealing system runs into the gap. At its end, the functional area for relubricating needs a covering for the gap that prevents the relubricated grease from exceeding from the sealing system on the wrong side.

If the sealing system can be supplied with fresh grease by another machine element like a bearing or gear next to the seal, the functional area for relubricating is not needed.

\section{Conclusions}

In the present work, the basic information from the literature about non-contacting sealing systems with grease-filled gaps are shown. The problem is that there are a lot of design examples but no information about how to choose the right sealing system and how to design a reliable sealing system according to the special working conditions. This was the beginning of the research project of Kümmel, which resulted in the development of guidelines to design a grease-filled labyrinth sealing 
system. Kümmel also showed that sealing of particles with those sealing systems is only possible if the grease-filled gap is open. An opened gap means that there are two layers of grease: one adhering at the static part, and one adhering at the rotating part of the sealing system. They are separated by a thin layer of air. This leads to very low losses caused by fluid friction.

The present work also describes the actual results of the research project to extend the guidelines of Kümmel to how to relubricate the sealing system. This is necessary if the surface of the grease layers inside the sealing system is saturated with particles. The result is that, compared with a fitting roller bearing, about three to five times the amount of grease is needed to relubricate the sealing system. If the grease loses a lot of its base oil, at least five times the amount of grease is needed. If the properties of the grease inside the sealing system undergo only little change, three times the amount of grease is sufficient to relubricate the sealing system.

It was observed that if there are particles bound by the grease, the ability to remove particles by relubricating the seal depends on the position of the gap in the sealing system. If the outer wall of the gap rotates, particles can hardly be removed by relubricating. If the inner wall of the gap rotates, particles can be removed by relubricating the sealing system, as shown in Figure 13. Because gaps with rotating inner and outer wall alternate, the sealing system can end after the first axial gap.

With additional experiments, the function of this sealing system with reduced complexity was investigated. The results show that the axial gap was free of particles after all experiments. Because of this, two functional areas were defined: sealing against particles and relubricating. If the functional area for relubricating the sealing system is not needed because the sealing system can be supplied with fresh grease by another machine element like a bearing or gear, the sealing system can only consist of a covered entrance area followed by a small axial gap.

The amount of particles that enter the sealing system depends on the operating condition of the sealing system. If the sealing system is heavily polluted with particles, the amount of particles that enter the sealing system during lifetime will be high. If the pollution of the sealing system is low to moderate, the amount of particles that enter the sealing system will be low. Because of this, the time between relubricating cycles can only be determined with experiments with the applied sealing system at respective operating conditions.

A large question concerns the opening of the gap. Although many experiments were evaluated, the mechanism leading to the opening of the gap is still unknown. Rheological properties of the grease are not sufficient to predict the opening of the gap. As is shown in this paper, the opening of the gap must additionally depend on the length of the gap. This must be investigated in further research projects and experiments.

Author Contributions: Experiments and article were done, analyzed and written by Florian Bosch and supervised by Werner Haas.

Conflicts of Interest: The authors declare no conflict of interest.

\section{References}

1. Bosch, F.; Haas, W. Relubricating of Grease Filled Labyrinth Seals; Poitiers-Futuroscope: Poitiers, France, 2016; pp. 42-52.

2. Bosch, F.; Haas, W. Sealing of Lubricating Grease without Friction with Non-Contacting Sealing Systems; Poitiers-Futuroscope: Poitiers, France, 2014; pp. 118-129.

3. Bosch, F.; Haas, W. Relubrication of Grease-Filled Labyrinth Sealing Systems. Konstruktion 2016, 68, 78-82. (In German)

4. Bosch, F.; Haas, W. Sealing of Dry Particles with Grease-Filled Labyrinth Sealing Systems; Arnold Tross Kolloquium: Hamburg, Germany, 2017. (In German)

5. Eschmann, P.; Hasbargen, L.; Weigand, K. The Practice of Rolling Bearing: Manual of the Calculation and Design of Bearings; R. Oldenbourg: München, Germany, 1953. (In German)

6. Schaeffler Technologies AG \& Co. KG: Wälzlagerpraxis. Handbuch zur Gestaltung und Berechnung von Wälzlagerungen, 4. Auflage, Mainz: Vereinigte Fachverlage, 2015, - ISBN 9783783004014. 
7. Dahlke, H. Manual of Rolling Bearing Practices; Part 2; Deutsche Koyo Wälzlager Verkaufsgesellschaft mbH: Hamburg, Germany, 1987. (In German)

8. Kümmel, J.; Haas, W. Grease-Filled Labyrinth Sealing Systems_Labyrinth Sealing Systems Filled with Grease to Seal Dust Particles, Abschlussbericht, Forschungsvorhaben; FKM Forschungskuratorium Maschinenbau e-V: Frankfurt/Main, Germany, 2010. (In German)

9. Bosch, F.; Kümmel, J.; Haas, W. Grease-filled labyrith sealing systems to seal dust and fluids. In Proceedings of the 17th ISC, Stuttgart, German, 13-14 September 2012. (In German)

10. Fritz, E.; Haas, W.; Müller, H.K. Non-Contacting Sealing Systems for Macine Tool Spindles—Design Guidelines and Solution Proposals; Berichte aus dem Institut für Maschinenelemente, Stuttgart; IMA: Stuttgart, Germany, 1991; Volume 39, ISBN 3-921920-39-6. (In German)

11. Stanger, N. Non-Contacting Sealing Systems for Small Installation Space. Ph.D. Thesis, Universität Stuttgart, Stuttgart, Germany, 1995; Volume 55, p. 111. (In German)

12. Baart, P.; Green, T.M.; Li, J.X.; Lundström, T.S.; Westerberg, L.G.; Höglund, E.; Lugt, P.M. The Influence of Speed, Grease Type, and Temperature on Radial Contaminant Particle Migration in a Double Restriction Seal. Tribol. Trans. 2011, 54, 867-877.

13. Baart, P. Grease Lubrication Mechanisms in Bearing Seals. Ph.D. Thesis, Luleå University of Technology, Luleå, Sweden, 2011; ISBN 978-91-7439-299-9.

14. NTN-SNR. Industry General Catalogue; TN04; Technical Catalogue: Annecy, France, 2009.

15. SKF GmbH. Rolling Bearings, Schweinfurt; PUB BU/P1 10000/3 EN; SKF Group: Gothenburg, Sweden, 2016.

16. FAG Kugelfischer Georg Schäfer AG. Rolling Bearing Lubrication; Publ. No. WL 81 115/4 EA; Industrial Bearings and Services: Green Bay, WI, USA, 2002.

17. Klüber Lubrication München KG. Isoflex TOPAS NCA 5051, Synthetic Long-Term Grease. In Product Information; Klüber Lubrication München KG: Munich, Germany, 17 August 2017.

18. Klüber Lubrication München KG. Klüberbio M 72-82. Readily Biodegradable Special Grease. In Product Information; Klüber Lubrication München KG: Munich, Germany, 17 August 2017.

19. Bartz, W.J. Lubricating Greases: Composition, Charactersistics, Testing and Application; Band 500, Kontakt \& Studium, Renningen-Malmsheim; Expert-Verlag: Renningen, Germany, 2000; ISBN 3-8169-1533-7. (In German) 